\title{
More to look in the diabetic patients: depression
}

\section{Editorial}

Five years ago, a very nice, 68years lady came to me with a long time history of hypertension and diabetes. Neither of them under good control. She was married, had 3 sons, and was in very good financial situation. Her BMI was $32 \mathrm{~kg} / \mathrm{m}^{2}$, blood pressure 150 X $100 \mathrm{mmHg}$ and the last Glycated Hemoglobin was of $11.9 \%$. A few months later she told me that she was having problems with her son and asked for an antidepressant. Sertraline, a selective serotonin reuptake inhibitor, was prescribed. After coming regularly for 2years, and changing her medication her blood pressure was under control and her A1C dropped to $7.2 \%$.

At that moment, when I thought everything was going well, I received a phone call informing that she committed suicide.

Suicide risk varies by age, sex, and race/ethnicity. Risk factors for suicide attempt include presence of a mental health disorder; serious adverse childhood events; family history of suicide; prejudice or discrimination associated with being lesbian, gay, bisexual, or transgender; access to lethal means; and possibly a history of being bullied, sleep disturbances, and chronic medical conditions. In men, socioeconomic factors, such as low income, occupation, and unemployment are also related to suicide risk. In older adults, additional risk factors, such as social isolation, spousal bereavement, neurosis, affective disorders, physical illness, and functional impairment, increase the risk for suicide. Risk factors of special importance to military veterans include traumatic brain injury, separation from service within the past 12 months, posttraumatic stress disorder, and other mental health conditions. Individual risk factors have only limited ability to predict suicide in an individual at any particular time. A large proportion of Americans have a risk factor for suicide; however, only a small proportion will attempt suicide, and even fewer will die from it. U.S. Prevention Services Task Force Organization (file:///Users/vivian/Downloads/suicidesumm.pdf October 2015).

Suicide in later life is a global public health problem, with those aged 65 and above constituting the demographic group with the highest suicide rate in most countries that report suicide statistics to the World Health Organization. ${ }^{1}$

\section{Age-group specific suicide rates have traditionally been highest}

Each year over 7,000 adults aged 60years and older die of suicide in the U.S., accounting for one of every five suicide deaths. Approximately $83 \%$ of those deaths between 2005 and 2007 were men, with a rate approximately six times that of women (27.0 and 4.5 per 100,000 populations, respectively). Centers for Disease Control and Prevention (CDC) Web-based Injury Statistics Query and Reporting System (WISQARS ${ }^{\mathrm{TM}}$ ) CDC; Atlanta, GA, USA: 2009. Available online: http://www.cdc.gov/ncipc/wisqars

Chronic medical condition, such as diabetes and hypertension may have emotional consequences that might be overlooked by the medical doctor who is taking care of the patient. Adaptation is sometimes complex since it involves some social restriction and lifestyle modification.
Volume 2 Issue 5 - 2015

\author{
Ellinger $V$ \\ Department of medicine, Universidade Severino Sombra, Brazil
}

Correspondence: Ellinger $\mathrm{V}$, Department of medicine, Universidade Severino Sombra, Brazil, Tel 552I 9912 452I, Email vivianellinger@gmail.com

Received: October 10, 2015 | Published: November 18, 2015

In a case control study Ceretta and collaborators ${ }^{2}$ evaluated, in 996 type 2 diabetic patients and 2.145 individuals without diabetes, the association of mood disorders, suicidal ideation and the quality of life in patients. The authors demonstrated a worse quality of life and a high comorbidity of type $2 \mathrm{DM}$ with depressive disorders and suicidal ideation.

Another study evaluated the mental health of patients with diabetes mellitus in Korea and compared it with mental health in the general Korean population. All data included in the final analyses were collected from 34,065 subjects (20years of age or older) who participated in the 2007-2012 Korea National Health and Nutrition Examination Survey (KNHANES). The mental health of 3846DM patients were compared with that of 30,219 controls. The report found that DM was associated with a marked increase in suicidal behaviors. ${ }^{3}$

\section{Another important point is the use of medication}

In a cohort study in the United Kingdom, the associations between different antidepressant treatments and the rates of suicide and attempted suicide or self harm in people with depression, showed an increased rate in the first 28days of starting and stopping antidepressants. The authors emphasize the need for careful monitoring of patients during these periods. ${ }^{4}$

Prior efforts to assess the impact of antidepressant use on risk of suicide attempt focused on antidepressant initiation or duration of use. Gaps remain in understanding risks associated with antidepressant discontinuation in the context of the drug regimen. Valuck et al. ${ }^{5}$ assessed the effects of antidepressant discontinuation on the risk of suicide attempt. A retrospective cohort of 2.4 million patients with depression, aged 5-89years, was created using standard Healthcare Effectiveness Data and Information Set (HEDIS) criteria; from this cohort, cases $(n=10,456)$ and controls $(n=41,815)$ were selected for study. Data were from a large, national, longitudinal, integrated claims database of managed care enrollees in the United States from calendar years 1999 through 2006. Antidepressant discontinuation showed a significant risk for suicide attempt, as did the period of an abbreviated trial, that is, stopping before a therapeutic regimen of 56days had been reached. The highest risk was associated with initiation, a finding consistent with other studies, closely followed by periods of dosing changes and discontinuation. Patients should be closely monitored during these periods. ${ }^{5}$

It is possible, that this was the case of this aforementioned patient, since she told me once, that she was taking "too many medicines". 


\section{Conclusion}

It is important for medical doctors treating patients with diabetes, to look not only at the Glycated Hemoglobin, but also to talk to the patient about depression and ask about suicide thoughts.

\section{Acknowledgements}

None.

\section{Conflict of interest}

Author declares that there is no conflict of interest.

\section{References}

1. World Health Organization. Suicide Prevention (SUPRE). 2015.
2. Ceretta LB, Réus GZ, Abelaira HM, et al. Increased prevalence of mood disorders and suicidal ideation in type 2 diabetic patients. Acta Diabetol. 2012;49(Suppl 1):S227-S234.

3. Chung JH, Moon K, Kim do H, et al. Suicidal ideation and suicide attempts among diabetes mellitus: the Korea National Health and Nutrition Examination Survey (KNHANES IV, V) from 2007 to 2012. J Psychosom Res. 2014;77(6):457-461.

4. Coupland C, Hill T, Morriss R, et al. Antidepressant use and risk of suicide and attempted suicide or self harm in people aged 20 to 64. cohort study using a primary care database. BMJ. 2015;350:h517.

5. Valuck RJ, Orton HD, Libby AM. Antidepressant discontinuation and risk of suicide attempt: a retrospective, nested case-control study. J Clin Psychiatry. 2009;70(8):1069-1077. 